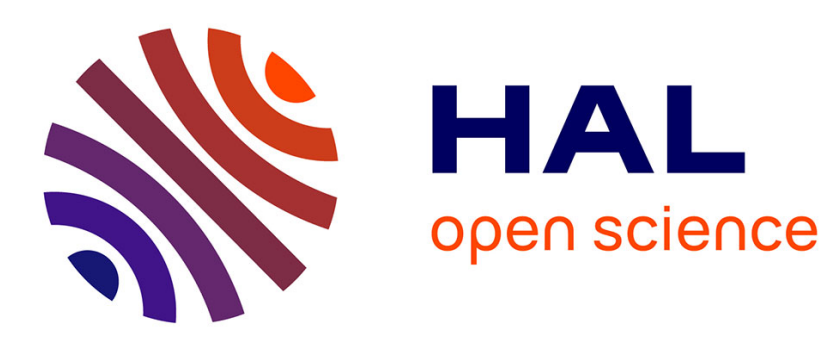

\title{
Is it time to reconcile HDL with cardiovascular diseases and beyond? An update on a paradigm shift
}

\author{
Laurent O Martinez, Cécile Ingueneau, Annelise Genoux
}

\section{To cite this version:}

Laurent O Martinez, Cécile Ingueneau, Annelise Genoux. Is it time to reconcile HDL with cardiovascular diseases and beyond? An update on a paradigm shift. Current Opinion in Lipidology, 2020, 31 (5), pp.302-304. 10.1097/MOL.0000000000000705 . inserm-03408018

\section{HAL Id: inserm-03408018 https://www.hal.inserm.fr/inserm-03408018}

Submitted on 28 Oct 2021

HAL is a multi-disciplinary open access archive for the deposit and dissemination of scientific research documents, whether they are published or not. The documents may come from teaching and research institutions in France or abroad, or from public or private research centers.
L'archive ouverte pluridisciplinaire $\mathbf{H A L}$, est destinée au dépôt et à la diffusion de documents scientifiques de niveau recherche, publiés ou non, émanant des établissements d'enseignement et de recherche français ou étrangers, des laboratoires publics ou privés. 


\title{
Is it time to reconcile HDL with cardiovascular diseases and beyond? An update on a paradigm shift
}

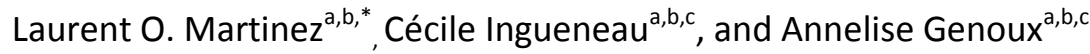

${ }^{a}$ Institut National de la Santé et de la Recherche Médicale (INSERM), UMR 1048, Institute of Metabolic and Cardiovascular Diseases, Toulouse, France,

${ }^{\mathrm{b}}$ University of Toulouse, UMR1048, Paul Sabatier University, Toulouse, France,

'Service de Biochimie, Pôle de biologie, Hôpital de Purpan, CHU de Toulouse, Toulouse, France,

* Corresponding author. INSERM UMR1048, 1 Avenue Jean Poulhes, BP 84225, 31432 Toulouse cedex 04, France. E-mail address: Laurent.Martinez@inserm.fr (L.O. Martinez)

Acknowledgements: None.

Financial support and sponsorship: None.

Conflicts of interest: There are no conflicts of interest 
According to numerous epidemiological studies, low plasma levels of high-density lipoprotein cholesterol (HDL-C) are independently associated with an increased risk of atherosclerotic cardiovascular disease (ASCVD). For instance, in patients with acute coronary syndrome (ACS), low HDL-C levels are associated with an increase in the number of recurrent cardiovascular events [1]. In addition, low plasma HDL-C levels also correlate with an increased risk of developing type 2 diabetes mellitus (T2DM), liver diseases, and dementia $\left[2,3^{*}, 4\right]$. However, although elevated HDL-C levels could be expected to be beneficial for health, randomized controlled trials of agents that increase HDL-C levels, such as cholesteryl ester transfer protein inhibitors (CETPi) and reconstituted HDL (rHDL), have failed to reveal a beneficial effect on cardiovascular disease outcomes $\left[5,6^{*}, 7\right]$. Moreover, Mendelian randomization analyses have reported that some genetic variants associated with elevated HDL-C levels are not associated with a lower risk of ASCVD, T2DM, or Alzheimer's disease $[8,9,10,11,12]$. However, the conclusions of these studies regarding the absence of a causal link between HDL-C levels and disease risk are questionable because the analyses did not take into account i) changes in HDL composition and functions in disease, ii) the complexity of HDL metabolism, as they excluded important genes from their analyses that regulate the levels of lipoproteins other than HDL-C [13].

These discordant results have led to the notion that beneficial functions of HDL particles are not determined solely by the cholesterol moiety in HDL (i.e., HDL-C). This paradigm shift has led to renewed interest in HDL research, particularly in terms of the identification of biomarkers that reflect causal structure-function-disease relationships of $\mathrm{HDL}$, with the ultimate goal of developing novel therapeutic approaches based on restoration/activation of HDL functions rather than increasing HDLC levels.

HDL particles exert pleiotropic atheroprotective activities, including cholesterol efflux from peripheral tissues to the liver for excretion by reverse cholesterol transport (RCT), in addition to exerting anti-infectious, antioxidative, anti-inflammatory, antithrombotic, anti-apoptotic, vasodilatory, and antidiabetic properties [14]. These pleiotropic beneficial activities rely on the fact that the plasma HDL fraction consists of heterogeneous and highly dynamic particles that contain hundreds of different quantitatively minor proteins and lipid species, most of which are thought to be biologically active $[6 *, 15]$.

Recently, analyses of data from large epidemiological population studies have revealed that the relationship between HDL-C levels and disease risks is nonlinear, with both extremely low and extremely high HDL-C levels associated with increased risks of ASCVD and infectious diseases $\left[6^{*}, 16,17,18\right]$. This observation could account for the negative results of trials of entities that enhance HDL-C levels. Further studies of this parabolic relationship have revealed that extremely 
high HDL-C levels (> $100 \mathrm{mg} / \mathrm{dL}$ ) are associated with endothelial dysfunction [19,20*], impaired glucose metabolism [21] and an impaired capacity of HDL to acquire free cholesterol from triglyceride-rich lipoprotein lipolysis [22]. Alternatively, extremely high HDL-C levels may be attributed to particular lifestyles such as excessive alcohol consumption, or genomic factors such as loss-of-function mutations in key HDL metabolism-regulating genes [23]. Thus, the identification of reliable markers for HDL functions requires in-depth knowledge of HDL complexity.

Under pathological conditions, quantitative and qualitative changes in the composition of HDL particles can compromise their beneficial properties for health [24**]. Interestingly, the distinct profile in the changes in HDL structure and lipid and protein compositions between coronary heart disease (CHD) and T2DM patients has led to the identification of three independent functional HDL components, namely the polyunsaturated sphingomyelin SM 42:3 and glycosylphosphatidylinositolphospholipase D1 (GPLD1), which inhibit endothelial cell apoptosis, and apolipoprotein F (apoF), which promotes maximal respiration of brown adipocytes [24**]. In terms of hepatic and cognitive diseases, apoA-I levels are negatively associated with the risks of chronic liver failure and dementia $\left[3^{*}, 4\right]$. Moreover, HDL particles that contain apoE but not apoJ may predict cognitive decline, independently of the presence of the apoE $\varepsilon 4$ allele [25]. Among the other emerging HDL-related biomarkers, ATPase inhibitory factor 1 (IF1) has been proposed to reflect hepatic HDL uptake (one of the last steps of RCT). IF1 has been reported to be independently and negatively associated with the long-term prognosis in CHD patients $[26,27]$. Importantly, a rapid and inexpensive high-throughput spectrometry-based assay that is amenable to clinical practice and that allows multiplexing in the quantification of circulating apolipoproteins and IF1 was recently developed and could serve to further validate a panel of these emerging HDL-related biomarkers [28,29*].

Studies of HDL-related biomarkers often comprise assessment of the Cholesterol Efflux Capacity (CEC) $\left[30^{* *}\right]$. A meta-analysis recently concluded that the CEC is negatively associated with cardiovascular morbidity and mortality [31]. However, as measuring CEC is time-consuming and it has not yet been standardized, there will need to be substantial technical progress before this biomarker will be used in routine clinical practice. A novel score was recently developed, called the HDL apolipoproteomic score ( $P C A D)$. This score, which correlates well with the CEC, is based on mass spectrometry quantification of specific HDL-associated apolipoproteins [32**]. Although the pCAD score warrants further clinical evaluation, it has been reported to be independently associated with cardiovascular mortality in CHD patients. Another clinically advanced HDL-related biomarker is based on measurement of the HDL particle number (HDL-P) by nuclear magnetic resonance spectroscopy. HDL-P has been shown to have a greater prognostic value than HDL-C and apoA-I in coronary patients [33], and it may be a surrogate marker of HDL anti-oxidative activity [30**]. 
Although further studies are needed to validate these biomarkers as proxies of the many protective properties of $\mathrm{HDL}$, their use, either alone or as part of a panel, should permit a biologicaland clinical-based reassessment of the optimal therapeutic strategy for targeting HDL. Thus, the use of HDL as therapeutics is contingent on further research, as indicated by the very limited number of current active clinical trials evaluating HDL-based therapies. For instance, of the three formulations of rHDL that have been developed for intravenous infusion, only a randomized controlled trial of the compound CSL-112 is still ongoing (expected completion in 2022), while the two other entities (MDCO-216 and CER-001) did not result in a reduction of atherosclerotic plaque in patients with acute coronary syndrome compared to placebo [30**]. rHDL formulations, which have exhibited disappointing outcomes to date, are mainly composed of poorly lipidated apoA-I and they are expected to exert a beneficial effect by increasing the CEC. However, it should be kept in mind that oxidative stress and inflammatory conditions, which occur in many chronic diseases, may convert HDL into dysfunctional particles. In particular, myeloperoxidase is enriched in atherosclerotic lesions and it promotes oxidative modification of apoA-I at residue Trp72, which is essential for the cholesterol efflux activity of apoA-I [34]. The control of the oxidative stress and inflammation status of patients should thus be evaluated to ensure that injected rHDL remain sufficiently functional to exert their intended beneficial effects. Alternatively, myeloperoxidase inhibitors could be used simultaneously to prevent lipoprotein oxidation [35]. Additionally, a short apoA-I-derived peptide was recently shown to improve glucose metabolism, promote cholesterol efflux, and reduce atherosclerosis in pre-clinical models, thus positioning this peptide as a promising drug candidate to reduce the risk of CVD in individuals with insulin resistance [36]. 


\section{REFERENCES AND RECOMMENDED READING}

Papers of particular interest, published within the annual period of review, have been highlighted as:

* of special interest

** of outstanding interest

1. Nakazawa M, Arashi H, Yamaguchi J et al. Lower levels of high-density lipoprotein cholesterol are associated with increased cardiovascular events in patients with acute coronary syndrome. Atherosclerosis 2020; 303:21-28.

2. Manandhar B, Cochran BJ, Rye KA. Role of High-Density Lipoproteins in Cholesterol Homeostasis and Glycemic Control. J Am Heart Assoc 2020; 9(1):e013531.

3. Trieb M, Rainer F, Stadlbauer V et al. HDL-related biomarkers are robust predictors of survival in patients with chronic liver failure. J Hepatol 2020; 73(1):113-120.

* This explorative cross-sectional study show that reduced levels of HDL-C and apoA-I are closely linked to the severity of liver failure, its complications and survival. The prognostic values of HDL-C and apoA-I were very similar to composite prognostic scores in patients with cirrhosis.

4. Button EB, Robert J, Caffrey TM et al. HDL from an Alzheimer's disease perspective. Curr Opin Lipidol 2019; 30(3):224-234.

5. Taheri H, Filion KB, Windle SB et al. Cholesteryl Ester Transfer Protein Inhibitors and Cardiovascular Outcomes: A Systematic Review and Meta-Analysis of Randomized Controlled Trials. Cardiology 2020; 145(4):236-250.

6. Jomard A, Osto E. High Density Lipoproteins: Metabolism, Function, and Therapeutic Potential. Front Cardiovasc Med 2020; 7:39.

* This comprehensive review outlines knowledge with respect to current understanding of HDLs behavior in health and diseases and summarize status of HDL-based therapies.

7. Riaz $\mathrm{H}$, Khan $\mathrm{SU}$, Rahman $\mathrm{H}$ et al. Effects of high-density lipoprotein targeting treatments on cardiovascular outcomes: A systematic review and meta-analysis. Eur J Prev Cardiol 2019; 26(5):533543.

8. Frikke-Schmidt R. HDL cholesterol and apolipoprotein A-I concentrations and risk of atherosclerotic cardiovascular disease: Human genetics to unravel causality. Atherosclerosis 2020; 299:53-55.

9. Karjalainen MK, Holmes M V., Wang $\mathrm{Q}$ et al. Apolipoprotein A-I concentrations and risk of coronary artery disease: A Mendelian randomization study. Atherosclerosis 2020; 299:56-63.

10. Haase CL, Tybjerg-Hansen A, Nordestgaard BG et al. HDL cholesterol and risk of type 2 diabetes: A mendelian randomization study. Diabetes 2015; 64(9):3328-3333.

11. Østergaard SD, Mukherjee S, Sharp SJ et al. Associations between Potentially Modifiable Risk Factors and Alzheimer Disease: A Mendelian Randomization Study. PLoS Med 2015; 12(6):e1001841.

12. Proitsi P, Lupton MK, Velayudhan L et al. Genetic Predisposition to Increased Blood Cholesterol and Triglyceride Lipid Levels and Risk of Alzheimer Disease: A Mendelian Randomization Analysis. PLoS Med 
2014; 11(9):e1001713.

13. Geller AS, Polisecki EY, Diffenderfer MR et al. Genetic and secondary causes of severe HDL deficiency and cardiovascular disease. J Lipid Res 2018; 59(12):2421-2435.

14. Kajani S, Curley S, McGillicuddy FC. Unravelling hdl-looking beyond the cholesterol surface to the quality within. Int J Mol Sci 2018; 19(7):1971.

15. Perségol L, Darabi M, Dauteuille C et al. Small dense HDLs display potent vasorelaxing activity, reflecting their elevated content of sphingosine-1-phosphate. J Lipid Res 2018; 59(1):25-34.

16. Zhong GC, Huang SQ, Peng Y et al. HDL-C is associated with mortality from all causes, cardiovascular disease and cancer in a J-shaped dose-response fashion: a pooled analysis of 37 prospective cohort studies. Eur J Prev Cardiol 2020; 0(0):1-17.

17. Madsen CM, Varbo A, Nordestgaard BG. Extreme high high-density lipoprotein cholesterol is paradoxically associated with high mortality in men and women: two prospective cohort studies. Eur Heart J 2017; 38(32):2478-2486.

18. Madsen CM, Varbo A, Tybjærg-Hansen A et al. U-shaped relationship of HDL and risk of infectious disease: two prospective population-based cohort studies. Eur Heart J 2018; 39(14):1181-1190.

19. Takaeko Y, Matsui S, Kajikawa M et al. Association of extremely high levels of high-density lipoprotein cholesterol with endothelial dysfunction in men. J Clin Lipidol 2019; 13(4):664-672.e1.

20. Chiesa ST, Charakida M, McLoughlin E et al. Elevated high-density lipoprotein in adolescents with Type 1 diabetes is associated with endothelial dysfunction in the presence of systemic inflammation. Eur Heart J 2019; 40(43):3559-3566.

* This cross-sectional study shows for the first time that early changes in renal function and chronic inflammation are associated with decreased HDL functionality in young people with Type 1 diabetes, and that these changes are related to early signs of vascular damage when HDL-C levels are also high.

21. Nakajima K, Higuchi R. Impaired Glucose Metabolism in People with Extremely Elevated High-Density Lipoprotein Cholesterol and Low Alcohol Consumption: Results of the Kanagawa Investigation of Total Checkup Data from the National Database-3 (KITCHEN-3). J Clin Med 2019; 8(11):1825.

22. Feng $M$, Darabi $M$, Tubeuf $E$ et al. Free cholesterol transfer to high-density lipoprotein (HDL) upon triglyceride lipolysis underlies the U-shape relationship between HDL-cholesterol and cardiovascular disease. Eur J Prev Cardiol 2019; 0(00):1-11.

23. Zanoni $\mathrm{P}$, Khetarpal SA, Larach DB et al. Rare variant in scavenger receptor $\mathrm{BI}$ raises HDL cholesterol and increases risk of coronary heart disease. Science 216; 351(6278):1166-1171.

24. Cardner $M$, Yalcinkaya $M$, Goetze $S$ et al. Structure-function relationships of HDL in diabetes and coronary heart disease. JCI Insight 2020; 5(1):e131491.

** This study shows that CHD and T2DM are associated with different changes in HDL protein and lipid composition. Although these observations cannot validate a causal relationship between the disease status and alterations of HDL, they served to elegantly identify and validate three novel determinants of HDL functions.

25. Koch M, DeKosky ST, Fitzpatrick AL et al. Apolipoproteins and Alzheimer's pathophysiology. Alzheimers Dement (Amst) 2018; 10:545-553. 
26. Martinez LO, Genoux A, Ferrières J et al. Serum inhibitory factor 1, high-density lipoprotein and cardiovascular diseases. Curr Opin Lipidol 2017; 28(4):337-346.

27. Genoux A, Lichtenstein L, Ferrières J et al. Serum levels of mitochondrial inhibitory factor 1 are independently associated with long-term prognosis in coronary artery disease: The GENES Study. BMC Med 2016; 14(1):125.

28. Genoux A, Duparc T, Ruidavets J-B et al. A reference measurement of circulating ATPase inhibitory factor 1 (IF1) in humans by LC-MS/MS: comparison with conventional ELISA. Talanta 2020. 219:121300.

29. Blanchard V, Garçon D, Jaunet C et al. A high-throughput mass spectrometry-based assay for large-scale profiling of circulating human apolipoproteins Running head: Multiplex assay for apolipoprotein quantification. J Lipid Res 2020. 61:1128-1139.

* This is the first validated LC-MS/MS method for the quantification of several apolipoproteins in a single run. The proposed method was multiplexed for the simultaneous analysis 18 apolipoproteins such as apoA-I, A-II, CI, CII, CIII, J, F and M.

30. Riggs KA, Rohatgi A. HDL and Reverse Cholesterol Transport Biomarkers. Methodist Debakey Cardiovasc J 2019; 15(1):39-46.

** Up-to-date and concise review summarizing knowledge with respect to HDL-related biomarkers and therapeutics.

31. Soria-Florido MT, Schröder H, Grau M et al. High density lipoprotein functionality and cardiovascular events and mortality: A systematic review and meta-analysis. Atherosclerosis 2020; 302:36-42.

32. Natarajan P, Collier TS, Jin Z et al. Association of an HDL Apolipoproteomic Score With Coronary Atherosclerosis and Cardiovascular Death. J Am Coll Cardiol 2019; 73(17):2135-2145.

** This article reports a significant advance in the development of a novel functional bioassay based on HDLassociated proteins.

33. Duparc T, Ruidavets J-B, Genoux A et al. Serum level of HDL particles are independently associated with long-term prognosis in patients with coronary artery disease: The GENES study. Sci Rep 2020; 10(1):8138.

34. Huang $\mathrm{Y}$, Didonato JA, Levison BS et al. An abundant dysfunctional apolipoprotein A1 in human atheroma. Nat Med 2014; 20(2):193-203.

35. Premkumar J, Sampath P, Sanjay R et al. Synthetic Guaiacol Derivatives as Promising Myeloperoxidase Inhibitors Targeting Atherosclerotic Cardiovascular Disease. ChemMedChem 2020; 15:1-14.

36. Edmunds SJ, Liébana-García R, Nilsson O et al. ApoAl-derived peptide increases glucose tolerance and prevents formation of atherosclerosis in mice. Diabetologia 2019; 62(7):1257-1267. 